\title{
Matrices cerámicas para la inmovilización de metales pesados sorbidos en cáscara de arroz
}

\section{Ceramic Matrices for Immobilization of Heavy Metals Sorbed on Rice Husk}

Presentación: 6/10/2020

\section{Doctorando:}

\section{Melisa Soledad Romano}

Grupo de Investigación de la Cáscara de Arroz (GICA), Facultad Regional Concepción del Uruguay, Universidad Tecnológica Nacional - Argentina

meliromano.06@gmail.com

\section{Director/es:}

\section{Ma. Del Carmen García}

Co-director/es:

\section{Nancy Quaranta}

\begin{abstract}
Resumen
Se han realizado numerosos estudios basados en la eliminación de iones de metales pesados de soluciones acuosas utilizando materiales lignocelulósicos, que son capaces de retener diversos contaminantes incluso presentes a bajas concentraciones. La cáscara de arroz constituye un residuo de biomasa que se ha estudiado en diversas aplicaciones. En años recientes se ha utilizado en procesos de biosorción de metales pesados. Después de este proceso, se genera un residuo biomasa-contaminante, que actualmente no tiene alternativas viables de bajo costo para su disposición final segura. Por lo tanto, el objetivo de este trabajo es evaluar la viabilidad de la incorporación a matrices cerámicas, de cáscara de arroz contaminada con $\mathrm{Cu}-\mathrm{Ni}-\mathrm{Cd}$ y $\mathrm{Pb}-\mathrm{Zn}$, con el fin de inmovilizar dichos metales pesados. Las piezas cerámicas se obtuvieron a partir de mezclas de arcilla comercial y cáscara de arroz con metal sorbido, añadidas en un $10 \%$ en volumen. Las materias primas se caracterizaron por diferentes técnicas, como SEM-EDS, XRD, DTA-TGA, distribución granulométrica, ecotoxicidad, etc., mientras que en los productos cerámicos obtenidos se analizó: porosidad, variación volumétrica permanente, ecotoxicidad, entre otros. Del análisis de los resultados, se puede concluir que las piezas inmovilizaron dentro de su estructura los metales contenidos en la cáscara de arroz agregada. Los ladrillos presentaron buenas propiedades físicas y mecánicas que hacen posible su uso en la construcción civil.
\end{abstract}

Palabras clave: Metales pesados, Cáscara de arroz, Matrices cerámicas, Ladrillos

\begin{abstract}
Numerous studies have been conducted about the removal of heavy metal ions from aqueous solutions using lignocellulosic materials, which are capable of adsorbing various contaminants present even at low concentrations.

Rice husk constitutes biomass waste that has found multiple applications in different fields, such as the removal of heavy metals by adsorption. After this process, a biomass-contaminant waste is generated, which currently does not have viable and low-cost alternatives for its final disposal. Thus, the objective of this work is to evaluate the possibility of incorporating rice husk contaminated with $\mathrm{Cu}-\mathrm{Ni}-\mathrm{Cd}$ and $\mathrm{Pb}-\mathrm{Zn}$ into ceramic matrices, to immobilize these hazardous metals. Ceramic pieces from mixtures of commercial clay and rice husk with adsorbed metal, added by $10 \%$ in volume were obtained. The raw materials were characterized by different techniques, such as SEM-EDS, XRD, DTATGA, particle size distribution, ecotoxicity, etc., while in ceramic products were analysed porosity, permanent volumetric variation, ecotoxicity, among others. From the overall results, it is possible to conclude that the obtained ceramic pieces have immobilized within their structure, the heavy metals present on rice husk. The bricks have excellent physical and mechanical properties that make possible their use in civil construction.
\end{abstract}


Keywords: Heavy metals, Rice husk, Ceramic matrices, Clay bricks

\section{Introducción}

La industrialización y el aumento poblacional han conducido a una gran demanda de productos y servicios, cuyos procesos generan, entre otros, efluentes líquidos con altas concentraciones de sustancias tóxicas bioacumulables, no biodegradables y cancerígenas. Estos efluentes deben tratarse adecuadamente para eliminar su toxicidad o reducirla a niveles adecuados para que puedan desecharse en los cursos de agua sin dañar los ecosistemas.

Los metales pesados, tales como plomo, cobre, cadmio, cinc y níquel, son considerados contaminantes ambientales prioritarios debido a la toxicidad que los iones metálicos presentan en los organismos vivos. Tras su liberación a partir de diversas fuentes, como pueden ser las actividades industriales y agrícolas, los metales pesados no son degradados y persisten en el medio ambiente (Tseveendorj et al., 2017). Actualmente se utilizan numerosas tecnologías para eliminar estos contaminantes de efluentes líquidos como la precipitación química, el intercambio iónico, los procesos de oxidación avanzados, la separación por membrana y la adsorción (Carolin et al., 2017). Estos tratamientos son costosos, producen residuos secundarios y, en muchos casos, no cumplen con los requisitos ambientales. En consecuencia, existe un gran interés en los sistemas de eliminación alternativos, como los procesos de biosorción, ya que han demostrado ser eficaces, económicos, versátiles y simples (Fomina \& Gadd, 2014; Park, Yun, \& Park, 2010).

Por otro lado, la agroindustria genera cantidades significativas de residuos de biomasa sin un uso específico. Hoy en día, se desarrollan numerosas investigaciones que estudian este tipo de desechos como biosorbentes de metales pesados (Abdolali et al., 2014; Neris et al., 2019). Sin embargo, no hay información científica sobre el tratamiento, la reutilización o el destino final de los nuevos residuos contaminantes de biomasa.

El arroz es uno de los cereales más consumidos en todo el planeta; cultivado en más de 100 países alrededor del mundo. Según la FAO, la producción anual mundial de arroz es mayor a 740 millones de toneladas y en Argentina es de aproximadamente 1.400.000 toneladas. El grano de arroz se compone principalmente de endospermo, cáscara, salvado y germen, en el que el endospermo representa el $70 \%$, la cáscara el 20-21\%, el salvado 6-8 \%, y el germen de arroz $1 \%$, respectivamente, del peso total de la semilla. Durante la producción de arroz se generan grandes cantidades de cáscaras que constituyen un residuo y un problema para las regiones productoras de este cereal (Zou \& Yang, 2019).

Por lo general, la cáscara es quemada o depositada en vertederos, con los correspondientes impactos ambientales que esto conlleva. El aprovechamiento de este material residual lograría mejorar la sostenibilidad de esta actividad agrícola y ayudar a reducir dichos impactos. La cáscara de arroz se ha utilizado en diferentes aplicaciones, entre las que se pueden citar, como combustible debido a su poder calorífico (Babaso \& Sharanagouda, 2017), como fuente de sílice y silicio debido al elevado contenido de $\mathrm{SiO}_{2}$ (Abbas et al., 2019; Almeida et al., 2019), para la preparación de carbón activado (Satayeva et al., 2018), en materiales de construcción (Muthuraj et al., 2019), y como adsorbente en el tratamiento de aguas (Palagama et al., 2019). En este último caso, luego de la retención de los contaminantes sobre la cáscara, se genera un residuo biomasa-contaminante, que actualmente no cuenta con alternativas viables y de bajo costo para la disposición final.

En este contexto, este trabajo evalúa la factibilidad de incorporación del residuo cáscara de arroz-contaminante en matrices cerámicas arcillosas para inmovilizar los metales pesados plomo, cinc, níquel, cobre, cadmio contenidos en estos materiales. Con este fin, la cáscara de arroz fue estudiada, en una primera instancia, en experimentos de adsorción, asegurando así que retenga una determinada cantidad de los contaminantes.

\section{Desarrollo}

La cáscara de arroz (CA) se obtuvo de una cooperativa de la provincia de Entre Ríos, luego del descascarillado del grano de arroz. Dado que las modificaciones químicas de la superficie del biomaterial pueden mejorar su capacidad de adsorción, la cáscara se trató con hidróxido de potasio (CA-KOH). El material natural se mezcló con KOH 1 M (relación 1:10) y se hirvió durante $30 \mathrm{~min}$. Se dejó en reposo durante una noche, se filtró y lavó con agua destilada y ácido clorhídrico al 2,5 M para alcanzar un pH de 5. Finalmente, se secó en estufa a $70^{\circ} \mathrm{C}$ por $24 \mathrm{~h}$.

Para determinar las condiciones óptimas del proceso de sorción, se analizaron varios factores, como el pH, el tiempo de contacto, la concentración inicial de $\mathrm{M}^{2+}$ y la masa de sorbente. Los ensayos de sorción fueron realizados en 
condiciones batch. Para ello, una determinada masa de biosorbente fue puesta en contacto con una solución de concentración conocida de plomo, níquel, cinc, cobre, plomo o cadmio a un dado pH y la suspensión resultante se dejó agitando el tiempo correspondiente de manera de garantizar el equilibrio de sorción. Las condiciones experimentales que optimizaron el proceso fueron $\mathrm{pH}=5-6$; dosis de sorbente: $3 \mathrm{~g} / \mathrm{L}$ para $\mathrm{Cd}^{2+}, 4 \mathrm{~g} / \mathrm{L}$ para $\mathrm{Cu}^{2+}, 4 \mathrm{~g} / \mathrm{L} \mathrm{para} \mathrm{Ni}^{2+}, 2$ $\mathrm{g} / \mathrm{L}$ para $\mathrm{Pb}^{2+}, 3 \mathrm{~g} / \mathrm{L}$ para $\mathrm{Zn}^{2+}$; concentración de $\mathrm{M}^{2+}: 50 \mathrm{mg} / \mathrm{L}$; tiempo de contacto: $60 \mathrm{~min}$; temperatura: $20 \pm 4{ }^{\circ} \mathrm{C}$.

Finalizados los ensayos de sorción, las muestras fueron filtradas y se procedió a la cuantificación del metal residual en la solución recolectada. La determinación del metal remanente se realizó por espectroscopía de absorción atómica utilizando una llama de aire-acetileno a la longitud de onda característica para cada metal en estudio. Los resultados obtenidos demostraron que los porcentajes de eliminación de metales por $\mathrm{CA}-\mathrm{KOH}$ fueron significativamente más altos que los del material no tratado (datos no mostrados).

La cáscara cargada con los contaminantes fue sometida a un proceso de molienda y tamizado, utilizando para la conformación de los ladrillos aquella con granulometría inferior a $1 \mathrm{~mm}$. Las piezas cerámicas se obtuvieron mediante presión uniaxial a $25 \mathrm{MPa}$ de mezclas arcilla comercial-cáscara de arroz con metal adsorbido, adicionadas en $10 \%$ en volumen, con $8 \%$ en peso de agua, en moldes de $70 \mathrm{~mm}$ x $40 \mathrm{~mm}$, resultando en ladrillos con $15 \mathrm{~mm}$ de espesor. La decisión de usar $10 \%$ de residuo en las muestras fue tomada teniendo en cuenta resultados preliminares (Pelozo et al., 2018). Luego de secadas, las muestras fueron tratadas térmicamente a $1000{ }^{\circ} \mathrm{C}$ siguiendo curvas de cocción similares a las utilizadas por la industria cerámica. Con fines comparativos, se preparó una muestra de arcilla comercial sin residuo adicionado.

Las materias primas fueron caracterizadas utilizando microscopía electrónica de barrido (SEM), análisis dispersivo de energía de rayos X (EDS), distribución granulométrica, ecotoxicidad, lixiviados, mientras que los productos cerámicos se caracterizaron con técnicas tales como: porosidad, absorción de agua, variación volumétrica permanente (VPP), pérdida de peso por calcinación (PPC), resistencia a la flexión, lixiviados y ecotoxicidad.

Los análisis SEM se efectuaron con un microscopio electrónico de barrido FEI Inspect S50, con analizador dispersivo de energía (EDAX-Phoenix). El módulo de rotura se obtuvo en una máquina DIGIMESS Modelo TG100L, con capacidad máxima de $500 \mathrm{~kg}$. La velocidad del ensayo fue de $0,5 \mathrm{~mm} /$ minuto. Los valores de VPP y PPC fueron obtenidos midiendo las dimensiones y el peso de cada muestra antes y después del tratamiento térmico a $1000{ }^{\circ} \mathrm{C}$. La porosidad y absorción de agua fueron determinadas de acuerdo a la Norma ASTM C20-00.

Las pruebas de ecotoxicidad se realizaron según norma IRAM 29114. Primero, se ensayaron las muestras de CA$\mathrm{KOH}$ cargado con metales sorbidos y luego las piezas cerámicas obtenidas al reemplazar parte de la arcilla comercial con residuos. De esta manera, se buscó determinar la fijación de los metales en la matriz cerámica. Se evaluó el número de semillas germinadas y el alargamiento de la radícula, expresado como un porcentaje de inhibición del crecimiento de la radícula, con respecto a las muestras de referencia con agua destilada.

El procedimiento para la caracterización de los lixiviados generados por $\mathrm{CA}-\mathrm{KOH}-\mathrm{M}^{2}+$ y los ladrillos se llevó a cabo adoptando el método EPA 1310B. La concentración de metales pesados en líquido lixiviado se determinó mediante un espectrofotómetro de absorción atómica a la longitud de onda característica de cada metal.

\section{Resultados}

El resultado del análisis químico realizado mediante EDS a la arcilla comercial y la cáscara natural, expresado en \% en peso de cada elemento, se muestra en la Tabla 1. Como puede observarse los elementos mayoritarios en ambos materiales son carbono, oxígeno y silicio.

\begin{tabular}{|c|c|c|c|c|c|c|c|c|c|}
\hline & C & $\mathbf{O}$ & $\mathbf{N a}$ & $\mathbf{M g}$ & Al & Si & K & Ca & Fe \\
\hline CA [\%] & 29,7 & 38,4 & -- & -- & -- & 22,3 & 1,9 & 2,1 & 5,6 \\
\hline Arcilla [\%] & 15,6 & 40,7 & 1,1 & 1,3 & 8,7 & 23,8 & 2,0 & 1,3 & 5,5 \\
\hline
\end{tabular}

Tabla 1. Análisis químico de las materias primas utilizadas

En la Figura 1 se observa la microestructura de la arcilla comercial, la cáscara de arroz natural y activada con KOH, obtenidas mediante SEM. La arcilla presenta una estructura homogénea de partículas de tamaño fino y partículas de mayor tamaño formadas por aglomerados de las más pequeñas (Fig. 1a). La microestructura de la CA natural es globular, mostrando un patrón celular del epicarpio o capa más externa, bien organizado, de estructura homogénea. 
Además, se observan estructuras alargadas aciculares, típicas de las estructuras fibrosas de ciertos cereales. En las muestras de $\mathrm{CA}-\mathrm{KOH}$ se observa un desprendimiento de estas estructuras desde el nacimiento de las mismas.

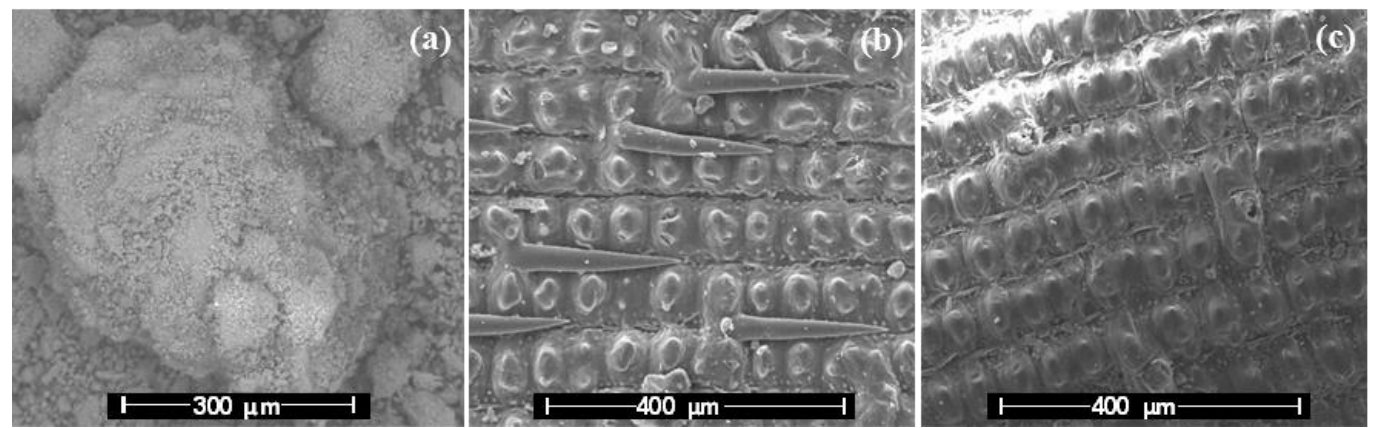

Figura 1: Imágenes SEM de (a) arcilla comercial, (b) CA natural, (c) CA- KOH. Magnificación: 500x.

Finalizadas las experiencias de sorción en las condiciones de proceso óptimas para cada metal divalente, se calculó la cantidad de metal retenido por CA-KOH. Estos resultados se observan en la Tabla 2.

\begin{tabular}{|c|c|c|c|c|c|}
\hline & $\mathbf{P b}^{\mathbf{2 +}}$ & $\mathbf{Z n}^{\mathbf{2 +}}$ & $\mathbf{N i}^{\mathbf{2 +}}$ & $\mathbf{C u}^{\mathbf{2 +}}$ & $\mathbf{C d}^{\mathbf{2 +}}$ \\
\hline $\mathbf{q}_{\mathrm{e}}\left[\mathbf{m g ~ M} \mathbf{~}^{\mathbf{2 +}} / \mathbf{g} \mathbf{C A}-\mathbf{K O H}\right]$ & $16,09 \pm 0,14$ & $9,83 \pm 0,39$ & $6,89 \pm 0,39$ & $7,81 \pm 0,40$ & $7,66 \pm 0,06$ \\
\hline
\end{tabular}

Tabla 2. Cantidad sorbida de cada metal sobre $\mathrm{CA}-\mathrm{KOH}$

A partir de los resultados de adsorción se determinaron las mezclas arcilla-cáscara de arroz contaminada para realizar las piezas cerámicas quedando para la conformación de las muestras, por un lado, aquellas contaminadas con plomo y cinc, y por otro, las contaminadas con níquel, cobre y cadmio. Se seleccionaron los metales de esta manera ya que las capacidades de retención en estos grupos fueron similares.

Los metales pesados sorbidos en $\mathrm{CA}-\mathrm{KOH}$ no se detectaron por EDS, posiblemente debido a su baja concentración. Por lo tanto, para determinar la presencia de contaminantes en $\mathrm{CA}-\mathrm{KOH}$, se realizó una prueba de lixiviación en el material cargado con los contaminantes metálicos. Los resultados se presentan en la Tabla 3.

\begin{tabular}{|l|c|c|c|c|c|}
\hline & Ni & Cu & Cd & Pb & Zn \\
\hline Concentración [mg/L] & 38,5 & 19,8 & 19,2 & 1,5 & 66,1 \\
\hline
\end{tabular}

Tabla 3. Análisis de lixiviados de CA-KOH luego de los ensayos de sorción

Teniendo en cuenta las Tablas 2 y 3 , es posible observar que el $\mathrm{Pb}^{2+}$, que registró la mayor cantidad sorbida por gramo de biomasa tratada, en las pruebas de lixiviado mostró las concentraciones más bajas. Esto podría indicar que su retención en la biomasa es excelente, del tipo de sorción química. Por otro lado, el ion $\mathrm{Zn}^{2+}$ fue el que presentó menor retención, probablemente debido a un proceso de sorción física. Con el mismo criterio, se consiguió concluir que los iones $\mathrm{Cd}^{2+} \mathrm{y} \mathrm{Cu}^{2+}$ exhibieron comportamientos similares, tanto en adsorción como en lixiviación, mientras que el ion $\mathrm{Ni}^{2+}$, que se comportó de manera similar a estos en la adsorción, se lixivió más fácilmente.

La prueba de ecotoxicidad determina la toxicidad de los químicos solubles en agua para las semillas de ciertas especies. La evaluación del efecto sobre el alargamiento de la radícula de las plántulas permite determinar el efecto tóxico de los compuestos solubles, presentes en una concentración tan baja que no son suficientes para inhibir la germinación, pero que, sin embargo, pueden retrasar o inhibir completamente los procesos de alargamiento de la radícula. Los resultados de esta prueba realizada a la biomasa contaminados con $\mathrm{Ni}$, $\mathrm{Cu}$ y $\mathrm{Cd}$ (CANCC) por un lado y contaminados con $\mathrm{Pb}$ y Zn (CAPZ) por el otro, a diferentes concentraciones, se incorporaron con fines comparativos a los resultados de la prueba de ecotoxicidad realizada en los ladrillos cerámicos obtenidos (Figura 3). Se observó que al aumentar la concentración del elutriado, aumentó la inhibición en el crecimiento de la radícula.

En la Figura 2 se muestra la apariencia macroscópica de los ladrillos obtenidos con arcilla comercial (AC) y con la adición de $10 \%$ en volumen de CA-KOH con Ni-Cu-Cd (BNCC) por un lado y Pb-Zn (BPZ), para el otro. Puede observarse que los productos presentan una coloración homogénea y una estructura bien definida sin desgranamiento de la misma. 


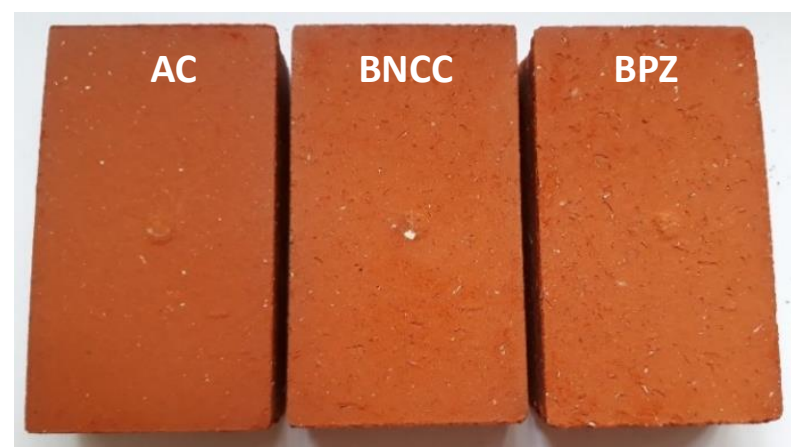

Figura 2: Apariencia de los ladrillos obtenidos.

Las propiedades analizadas en los productos cerámicos obtenidos: PPC, VVP, porosidad, absorción de agua y módulo de rotura, se muestran en la Tabla 4. Las muestras con CA presentan valores de pérdida de peso por calcinación superiores (mayor al 50\%) en comparación con las muestras de arcilla comercial, sin agregado de residuo. En cambio, la variación volumétrica permanente de todas las muestras se encuentra alrededor del mismo rango, presentando una diferencia del $10 \%$ en la muestra CAPZ con respecto a la de arcilla comercial. Este hecho puede explicarse debido a que durante el proceso de cocción de los ladrillos se produce la combustión de la CA generando poros en la estructura. Esto queda demostrado en los valores de porosidad de las muestras donde se ve un incremento de hasta el $20 \%$ en la muestra CANCC. En cambio, la resistencia a la flexión es menor en las muestras con residuo agregado. Este comportamiento está relacionado con el aumento en la porosidad que afecta la cohesión y reduce la resistencia del material a la fractura. Las características de las piezas cerámicas obtenidas se encuentran dentro de los valores establecidos por el mercado y los establecidos por la norma ASTM C410-60 para ladrillos para piso industrial.

\begin{tabular}{|l|c|c|c|}
\hline & AC & BNCC & BPZ \\
\hline PPC [\%] & 5,2 & 8,7 & 8,4 \\
\hline VVP [\%] & $-10,6$ & $-10,0$ & $-9,6$ \\
\hline Porosidad [\%] & 29,0 & 35,0 & 32,0 \\
\hline Absorción de agua [\%] & 16,0 & 21,0 & 18,0 \\
\hline MOR [MPa] & 7,0 & 5,2 & 5,2 \\
\hline
\end{tabular}

Tabla 4. Propiedades de los productos cerámicos obtenidos

La Figura 3 muestra los resultados de las pruebas de ecotoxicidad realizadas en ladrillos cerámicos. Se observó que, para bajas concentraciones, los porcentajes de inhibición del crecimiento de la raíz no difieren significativamente de los obtenidos en CA-KOH con metales sorbidos. Sin embargo, a partir de niveles más altos (50\%), el efecto inhibidor de los ladrillos es menor en comparación con el obtenido en la cáscara de arroz contaminada.

Por otro lado, el análisis de los líquidos lixiviados de las piezas cerámicas con CA-KOH se realizó empleando espectroscopía de absorción atómica. Los valores medidos se hallaron por debajo del límite de detección del método y, en consecuencia, los niveles de los contaminantes analizados están por debajo de los valores establecidos por la normativa vigente.

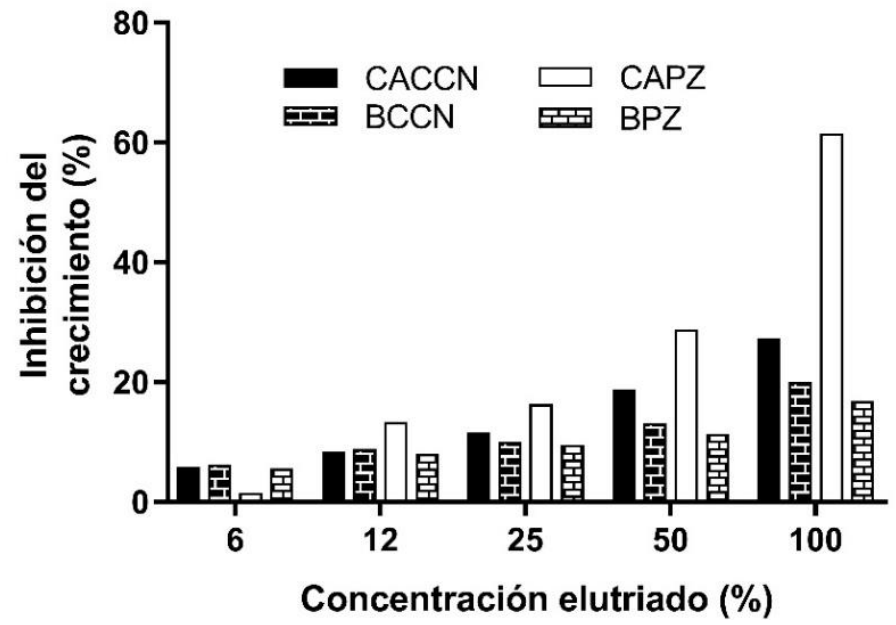

Figura 3: Análisis de ecotoxicidad para $\mathrm{CA}-\mathrm{KOH}-\mathrm{M}^{2+}$ y para los ladrillos obtenidos. 
Los resultados de las pruebas de ecotoxicidad y lixiviación realizadas en los productos finales sugieren la viabilidad de incorporar cáscaras de arroz con metales pesados adsorbidos, en matrices cerámicas para lograr la fijación de estos por la estructura.

\section{Conclusiones}

En este trabajo se evaluó la factibilidad de incorporación del residuo cáscara de arroz-contaminante en matrices cerámicas arcillosas para inmovilizar los metales pesados plomo, cinc, níquel, cobre, cadmio contenidos en las cáscaras. A partir de los resultados obtenidos puede concluirse que las piezas cerámicas obtenidas han inmovilizado dentro de su estructura los metales pesados adsorbidos en las cáscaras de arroz agregadas. Además, presentan una estructura definida sin desgranamientos con tonalidad homogénea, bordes definidos, buen grado de sinterización y propiedades adecuadas para su uso en servicio.

\section{Referencias}

Abbas, N., Khalid, H. R., Ban, G., Taik, H., y Lee, H. K. (2019). Silica aerogel derived from rice husk: an aggregate replacer for lightweight and thermally insulating cement-based composites. Construction and Building Materials, 195, 312322. doi:10.1016/j.conbuildmat.2018.10.227

Abdolali, A., Guo, W. S., Ngo, H. H., Chen, S. S., Nguyen, N. C., y Tung, K. L. (2014). Typical lignocellulosic wastes and byproducts for biosorption process in water and wastewater treatment: A critical review. Bioresource Technology, 160, 57-66. doi:10.1016/j.biortech.2013.12.037

Almeida, S. R., Elicker, C., Vieira, B. M., Cabral, T. H., y Silva, A. F. (2019). Black $\mathrm{SiO}_{2}$ nanoparticles obtained by pyrolysis of rice husk. Dyes and Pigments, 164, 272-278. doi:10.1016/j.dyepig.2019.01.030

Babaso, P. N., y Sharanagouda, H. (2017). Rice Husk and Its Applications: Review. International Journal of Current Microbiology and Applied Sciences, 6(10), 1144-1156. doi:10.20546/ijcmas.2017.610.138

Carolin, C. F., Kumar, P. S., Saravanan, A., Joshiba, G. J., y Naushad, M. (2017). Efficient techniques for the removal of toxic heavy metals from aquatic environment: A review. Journal of Environmental Chemical Engineering, 5(3), 27822799. doi:10.1016/j.jece.2017.05.029

Fomina, M., y Gadd, G. M. (2014). Biosorption: current perspectives on concept, definition and application. Bioresource Technology, 160, 3-14. doi:10.1016/j.biortech.2013.12.102

Muthuraj, R., Lacoste, C., Lacroix, P., y Bergeret, A. (2019). Sustainable thermal insulation biocomposites from rice husk, wheat husk, wood fibers and textile waste fibers: Elaboration and performances evaluation. Industrial Crops and Products, 135, 238-245. doi:10.1016/j.indcrop.2019.04.053

Neris, J. B., Luzardo, F. H. M., da Silva, E. G. P., y Velasco, F. G. (2019). Evaluation of adsorption processes of metal ions in multi-element aqueous systems by lignocellulosic adsorbents applying different isotherms: A critical review. Chemical Engineering Journal, 357, 404-420. doi:10.1016/j.cej.2018.09.125

Palagama, D. S. W., Devasurendra, A. M., Baliu-Rodriguez, D., Kirchhoff, J. R., y Isailovic, D. (2019). Treated rice husk as a recyclable sorbent for the removal of microcystins from water. Science of the Total Environment, 666, 1292-1300. doi:10.1016/j.scitotenv.2019.02.042

Park, D., Yun, Y.-S., y Park, J. M. (2010). The past, present, and future trends of biosorption. Biotechnology and Bioprocess Engineering, 15(1), 86-102. doi:10.1007/s12257-009-0199-4

Pelozo, G., Quaranta, N., Caligaris, M., Romano, M., y Cristóbal, A. (2018). Effect of the Incorporation of Biomass Wastes on the Properties of Fired Clay Bricks. In 33rd International Conference on Solid Waste Technology and Management (pp. 744-751). Annapolis, Washington DC, U.S.A.: Ed. Widener University.

Satayeva, A. R., Howell, C. A., Korobeinyk, A. V., Jandosov, J., Inglezakis, V. J., Mansurov, Z. A., y Mikhalovsky, S. V. (2018). Investigation of rice husk derived activated carbon for removal of nitrate contamination from water. Science of the Total Environment, 630, 1237-1245. doi:10.1016/j.scitotenv.2018.02.329

Tseveendorj, E., Enkhdul, T., Lin, S., Dorj, D., Oyungerel, S., y Soyol-Erdene, T. O. (2017). Biosorption of lead (II) from an aqueous solution using biosorbents prepared from water plants. Mongolian Journal of Chemistry, 18(44), 5261. doi:10.5564/mjc.v18i44.937

Zou, Y., y Yang, T. (2019). Rice Husk, Rice Husk Ash and Their Applications. In C. Ling-Zh \& X. Xuebing (Eds.), Rice Bran and Rice Bran Oil (pp. 207-246). Elsevier Inc. doi:10.1016/b978-0-12-812828-2.00009-3 